\title{
Dielectric and Piezoelectric Properties of Nylon 9 and Nylon 11
}

\author{
Gang Wu, Okimichi YaNO, and Toshiichi SoEN \\ Department of Polymer Science and Engineering, Kyoto Institute of Technology, \\ Matsugasaki, Kyoto 606, Japan
}

(Received April 5, 1985)

\begin{abstract}
Various specimens of nylon 9 and nylon 11 were prepared, and their crystalline structures, the temperature and frequency dependences of electric properties were investigated. Dielectric constants (at $10 \mathrm{~Hz}$ ) of various specimens were about $2.5-3$ in the temperature range from $-160^{\circ} \mathrm{C}$ to room temperature, and they began to increase near $T_{\mathrm{g}}$ and reached above 30 at about $100^{\circ} \mathrm{C}$. They showed three dielectric loss peaks due to two types of local mode motion and the glass transition. Piezoelectric behavior of the $\alpha$ form specimen obtained by slow cooling of melts and that of the $\gamma$ form specimen obtained by casting from its trifluoroacetic acid solution were almost the same, but a mixture of $\alpha$ and $\gamma$ forms obtained by quenching of the melts into liquid nitrogen had a larger piezoelectric constant than the above two. From these results it may be considered that the lower regularity in the dipole orientation within the mixture makes it relatively easier to rearrange the dipoles in parallel to the electric field in the poling process. Nylon 9 had a larger piezoelectric constant than nylon 11 , which may be due to the higher dipole density of nylon 9. The piezoelectric stress constant of the stretched film was larger than the unstretched one, but the piezoelectric strain constant of both were similar. The largest piezoelectric stress constant of $2.1 \mathrm{mCm}^{-2}$ (at $25^{\circ} \mathrm{C}$ ) was obtained from a stretched nylon 9 specimen and the largest piezoelectric strain constant of $1.1 \mathrm{pCN}^{-1}\left(\right.$ at $\left.25^{\circ} \mathrm{C}\right)$ was obtained for nylon 9 specimen which was a mixture of $\alpha$ and $\gamma$ forms.
\end{abstract}

KEY WORDS Odd Nylons / Nylon 9 / Nylon 11/ Dielectric Property /

Piezoelectric Property /

Dielectric and piezoelectric properties of polymers are closely related to the chemical and crystalline structure of materials and the dipole density in the crystalline unit cell. The all trans conformation of the odd nylon molecules such as nylon 9 and nylon 11 gives a large dipole moment perpendicular to the chain axis and all dipole moments are aligned to the same direction. So the odd nylons can have a spontaneous polarization in the unit cell of the crystalline phase, and thus the odd nylons are good piezoelectric materials.

It has been reported that nylon 11 has a triclinic $\alpha$ form and a pseudohexagonal $\gamma$ form. ${ }^{1}$ The $\alpha$ form can be obtained by slowly cooling the melt to room temperature, and the $\gamma$ form by quenching from the melt. ${ }^{2,3}$ The electric properties of these nylon 11 specimens of $\alpha$ and $\gamma$ phases have been also reported; ${ }^{2-7}$ under equivalent poling conditions, the piezoelectric constants obtained from $\gamma$ structure are higher than that of $\alpha$ structure. It is proposed that the field-induced dipole alignment might more effectively occur in the $\gamma$ structure than in the ordinary $\alpha$ structure.

To the best of our knowledge, no report has been published about the dielectric and piezoelectric properties of nylon 9. According to the method of ref. 4 , the dipole density in the crystal phase of nylon 9 is estimated to be higher than that of nylon 11. Therefore nylon 9 may be expected to show a larger piezoelectric effect than nylon 11. In this work, we prepared various specimens of 
nylon 9 and nylon 11, and compared their electrical properties.

\section{EXPERIMENTAL}

The pellets of nylon 9 and nylon 11 were provided from Popular Plastic Corporation (Huaiyin, China) and General Science Corporation, respectively. Films were made from the pellets by various methods as shown in Table I. The $\alpha$ form specimens ( $\alpha 9$ and $\alpha 11$ ) were obtained by melting the pellets and slowly cooling to room temperature. The $\gamma$ form specimens $(\gamma 9$ and $\gamma 11)$ were obtained by casting from their trifluoroacetic acid solutions and dried for about $12 \mathrm{~h}$ under vacuum at $75^{\circ} \mathrm{C}$. The pure $\gamma$ form specimen could not be obtained by the quenching method reported in ref 2 and 3. The mixtures of $\alpha$ and $\gamma$ forms (M9 and M11) were also used in this work, which were obtained by quenching the melts into liquid nitrogen. These specimens (M9 and M11) were uniaxially stretched about four times in an oil bath at $175^{\circ} \mathrm{C}$ for nylon 9 and at $160^{\circ} \mathrm{C}$ for nylon 11 , and then slowly cooled to room temperature. The obtained specimens were coded as S- $\alpha 9$ and $S-\alpha 11$. The oriented $\gamma$ form films (S- $-\gamma 9$ and $S-\gamma 11$ ) were obtained by treating the S$\alpha 9$ and $S-\alpha 11$ specimens with an iodine-potassium iodide aqueous solution. After the treatment the adsorbed iodine was removed by a sodium sulfate aqueous solution.

The dielectric measurement was carried out by the three-terminal electrode method. The surfaces of the specimens were coated with pure silver by the vacuum evaporation method, and then used for the dielectric measurement. Each specimen was set in a sealed cell and dried under vacuum $\left(10^{-3}\right.$ Torr $)$ at $80^{\circ} \mathrm{C}$ for $12 \mathrm{~h}$ in advance. Dry nitrogen gas was then introduced into the cell. The dielectric constant was measured at the frequency of $10,30,100,300,1000,3000$, and $10000 \mathrm{~Hz}$ from $-160^{\circ} \mathrm{C}$ to $100^{\circ} \mathrm{C}$ with increasing temperature. For the piezoelectric measurement,
Table I. Preparation methods of various specimens

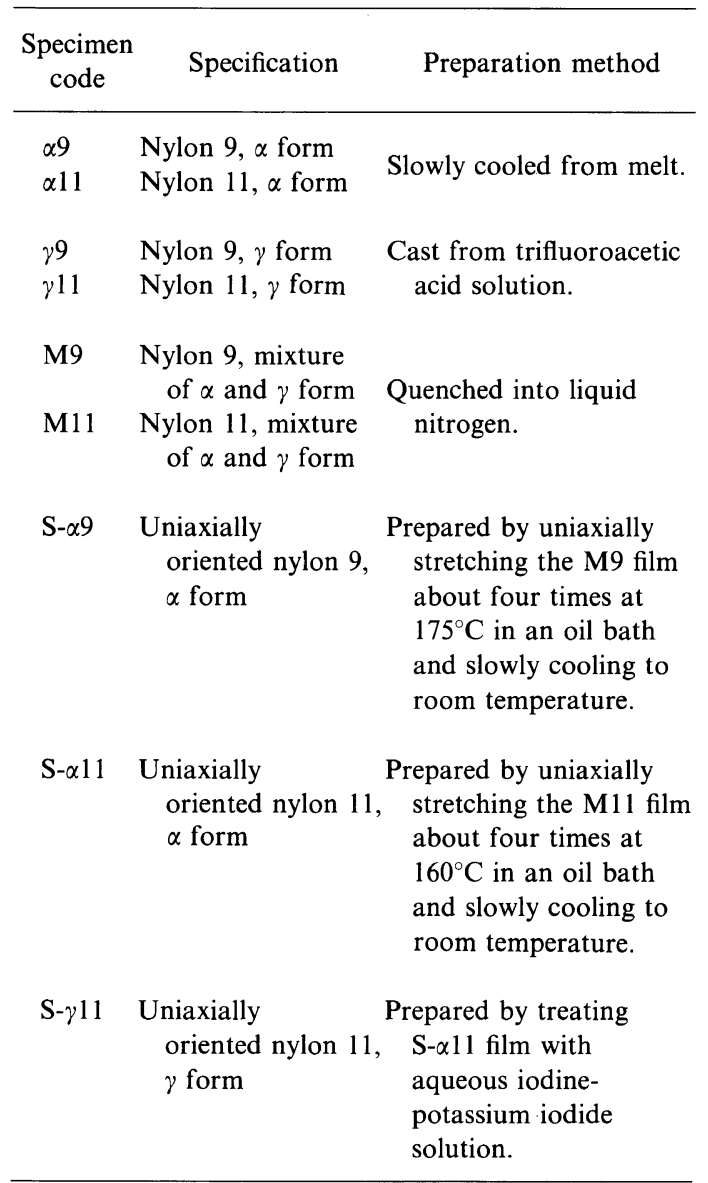

the coated specimens were dried at $10^{-3}$ Torr at $85^{\circ} \mathrm{C}$ for $2 \mathrm{~h}$, poled in an electric field of $400 \mathrm{kV} \mathrm{cm}^{-1}$ at the same pressure at $80^{\circ} \mathrm{C}$ for $15 \mathrm{~min}$, and cooled in the same field to room temperature. Both the piezoelectric strain constant $d_{31}^{\prime}$ and the piezoelectric stress constant $e_{31}^{\prime}$ were measured by the direct method at $80 \mathrm{~Hz}$ from $-70^{\circ} \mathrm{C}$ to $100^{\circ} \mathrm{C}$.

\section{RESULTS AND DISCUSSION}

\section{Characterization of Specimens}

The identification of the specimens was carried out by X-ray diffraction and the infrared spectroscopy. Films of $\alpha 9$ and $\alpha 11$ forms showed ordinary X-ray diffraction patterns 


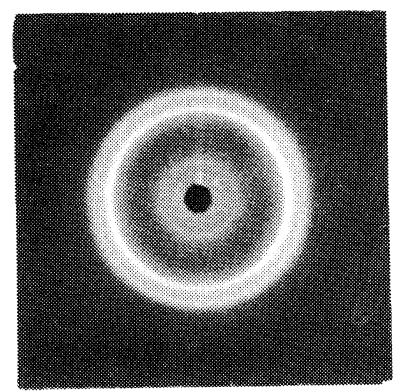

(a)

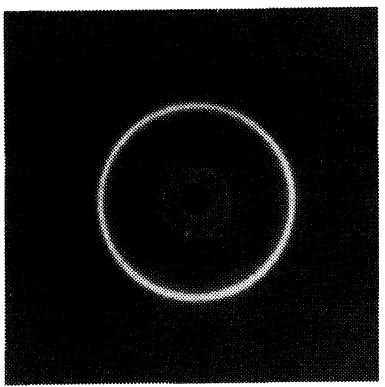

(b)

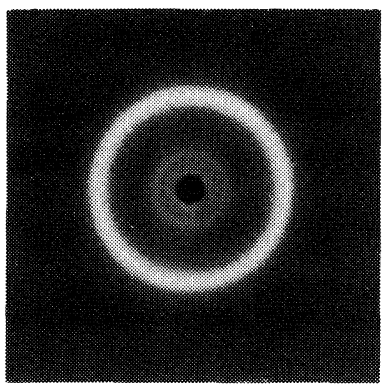

(c)

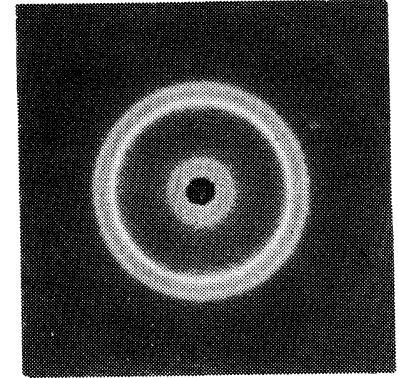

(d)

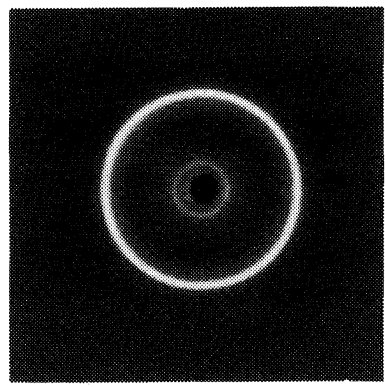

(e)

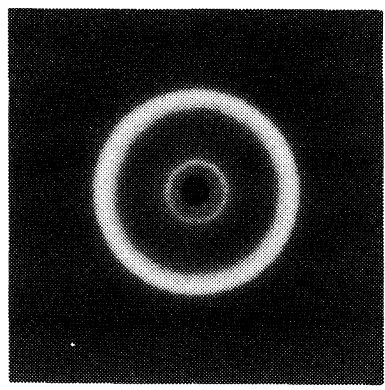

(f)

Figure 1. X-ray diffraction diagrams of the nylon 9 and nylon 11 films: (a) and (d), films slowly cooled from the melts ( $\alpha 9$ and $\alpha 11$ ); (b) and (e), films cast from the trifluoroacetic acid solution ( $\gamma 9$ and $\gamma 11$ ); (c) and (f), films quenched from the melts (M9 and M11).

of the triclinic form with three rings as shown in Figures 1a and 1d. The lattice spacings calculated from these diffraction patterns were $3.72,4.33$, and $9.77 \AA$ for nylon $9(\alpha 9)$ and $3.77,4.38$, and $11.9 \AA$ for nylon 11 $(\alpha 11)$. These values agree well with the lattice spacings for the $\alpha$ forms of nylon 9 and nylon $11 .^{8,9}$ On the other hand, the X-ray diffraction patterns of the $\gamma 9$ and $\gamma 11$ films cast from trifluoroacetic acid solutions showed two rings (Figures $1 \mathrm{~b}$ and $1 \mathrm{e}$ ). For the $\gamma 11$ film, the observed spacings of 4.08 


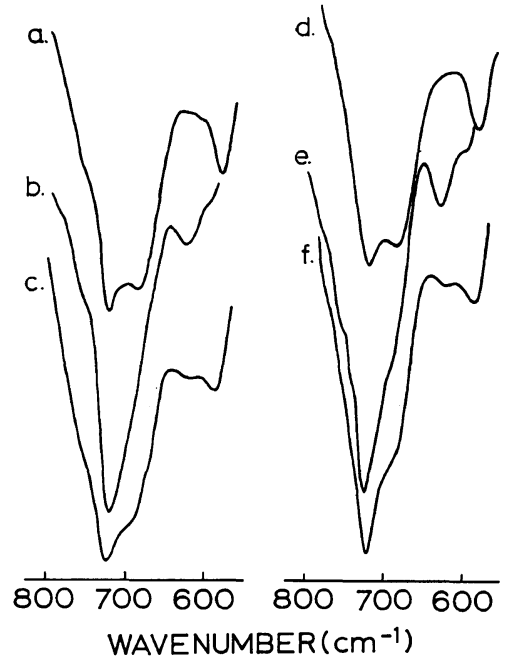

Figure 2. Infrared spectra of the nylon 9 and nylon 11 films: (a) and (d), films slowly cooled from the melts $(\alpha 9$ and $\alpha 11$ ); (b) and (e), films cast from the trifluoroacetic acid solution ( $\gamma 9$ and $\gamma 11)$; (c) and (f), films quenched from the melts (M9 and M11).

and $14.4 \AA$ correspond to the reported unit cell parameters of the $\gamma$ form of nylon $11 .{ }^{9}$ For the $\gamma 9$ film, the calculated spacings of the two rings were 4.07 and $11.8 \AA$, and its pattern was similar to that of the $\gamma$ form of nylon 11. So it seems that nylon 9 can be crystallized as the $\gamma$ form.

The films of the $\alpha$ and $\gamma$ forms showed different infrared spectra from each other as shown in Figure 2. In this figure, a, b, d, and e represent the spectra of $\alpha 9, \gamma 9 \quad \alpha 11$, and $\gamma 11$, respectively. A significant change was observed in the $690-580 \mathrm{~cm}^{-1}$ region. The amide $\mathrm{V}$ band ( $\mathrm{NH}$ out-of-plane vibration) ${ }^{10}$ at $687 \mathrm{~cm}^{-1}$ and the amide VI band at $584 \mathrm{~cm}^{-1}$ were observed in the infrared spectra of $\alpha 9$ and $\alpha 11$ films. On the other hand, in the spectra of $\gamma 9$ and $\gamma 11$ films, the above two absorptive bands disappeared but a new band appeared at $627 \mathrm{~cm}^{-1}$. These results agree well with those in the published report. ${ }^{9}$ The films quenched from the melt displayed a strong but diffused ring at about $4.11 \AA$ in the $\mathrm{X}$-ray diffraction patterns (Figures 1c and 1f) and showed the infrared

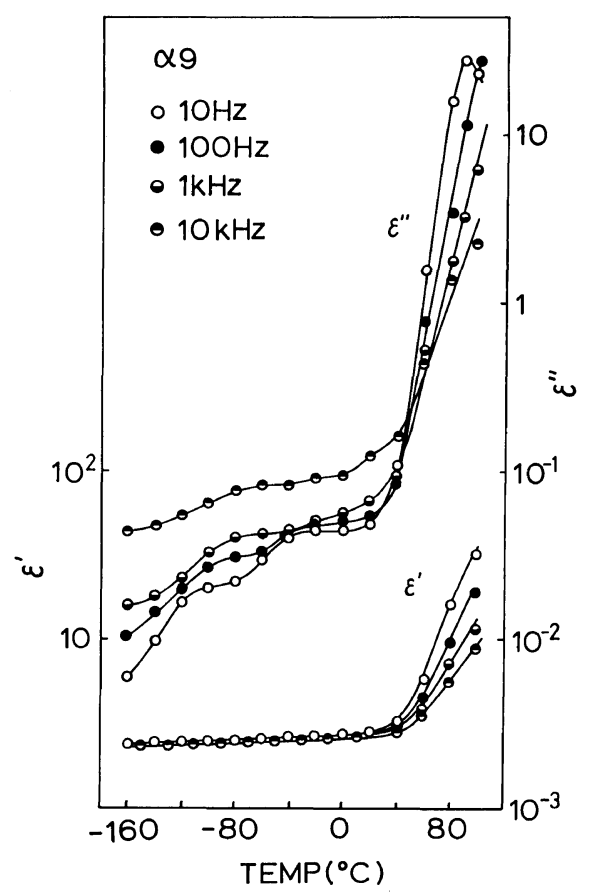

Figure 3. Temperature dependence of dielectric constants of $\alpha 9$ specimens.

spectra of an intermediate structure between the $\alpha$ and $\gamma$ forms (Figures 2c and 2f). From these results it was reasonable to consider that the quenched films are composed of the $\alpha$ and $\gamma$ forms.

\section{Dielectric Measurements}

Temperature Dependence of Dielectric Constants. Figure 3 shows the temperature dependence of real and imaginary parts of complex dielectric constants at 10,100,1000, and $10000 \mathrm{~Hz}$ for nylon 9. The dielectric constant $\varepsilon^{\prime}$ increased gradually with increasing temperature. Below $0^{\circ} \mathrm{C}$, it was relatively low and about $2.5-3$ at various frequencies. It increased rapidly as the temperature was raised from $20^{\circ} \mathrm{C}$ to $100^{\circ} \mathrm{C}$. The dielectric constant $\varepsilon^{\prime}$ increased to above 30 at about $100^{\circ} \mathrm{C}$ for $10 \mathrm{~Hz}$. Corresponding to such a temperature dependence of $\varepsilon^{\prime}$, the dielectric loss factor $\varepsilon^{\prime \prime}$ showed three loss peaks labelled as $\gamma, \beta$, and $\alpha$. The $\gamma$ peak due to the local 


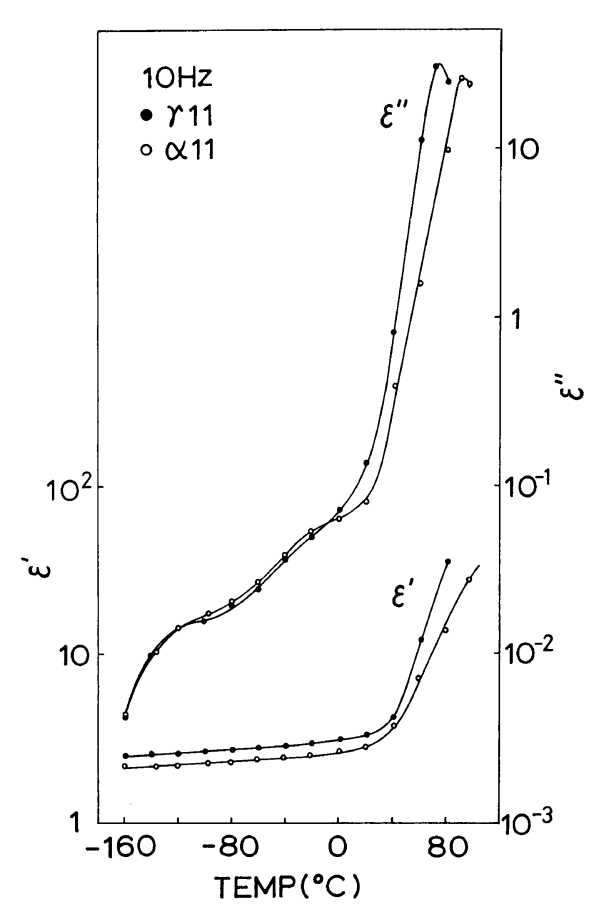

Figure 4. Temperature dependence of dielectric constants of $\alpha 11$ and $\gamma 11$ specimens.

mode motion appeared at about $-110^{\circ} \mathrm{C}$ at $10 \mathrm{~Hz}$. The $\beta$ peak around $-30^{\circ} \mathrm{C}$ at $10 \mathrm{~Hz}$ was related to the adsorbed water and motions of the dipolar amide groups. ${ }^{11}$ The value of $\varepsilon^{\prime \prime}$ increased rapidly about room temperature with increasing temperature and a large peak appeared about $90^{\circ} \mathrm{C}$. This corresponds to the primary dispersion, and is called the $\alpha$ relaxation. The locations of the above mentioned three peaks shifted to the higher temperature side as the frequency was increased.

The dielectric behavior of the $\alpha$ and $\gamma$ form films at $10 \mathrm{~Hz}$ is compared in Figure 4. The dielectric constant of the $\gamma 11$ specimen was higher than that of $\alpha 11$ at high temperature, and the primary dispersion peak of the $\gamma 11$ shifted to the lower temperature side. These characteristics were also observed in the cases of nylon 9 films ( $\alpha 9$ and $\gamma 9$ ), but the reasons are not clear at present.

Poling Effects. The $\alpha$ form films of nylon 9

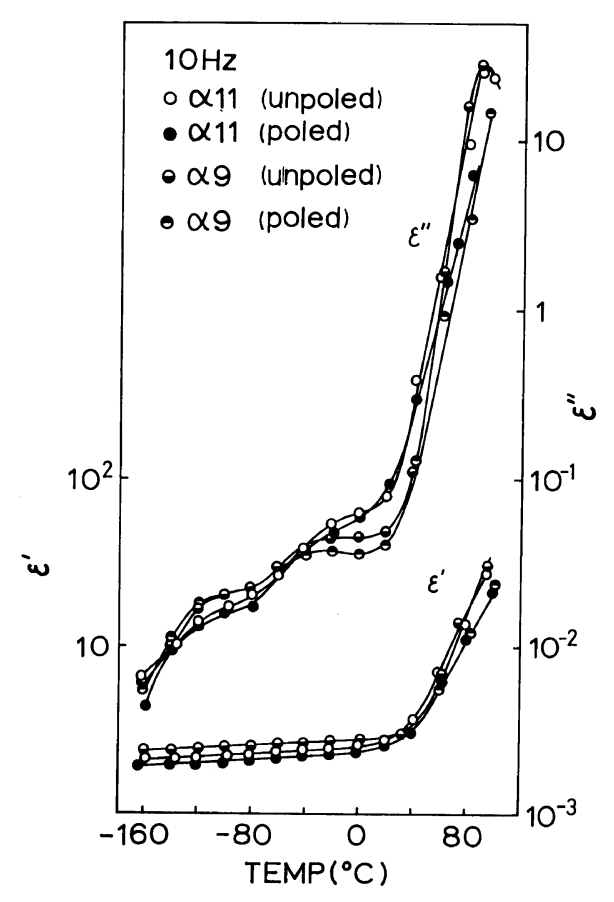

Figure 5. Temperature dependence of dielectric constants of poled and unpoled specimens.

and nylon 11 were poled at $300 \mathrm{kV} \mathrm{cm}^{-1}$ at $80^{\circ} \mathrm{C}$, and then their dielectric behavior was measured. The dielectric behavior of poled nylon 9 and nylon 11 at $10 \mathrm{~Hz}$ is shown in Figure 5 in comparison with that of unpoled ones. The dielectric constant $\varepsilon^{\prime}$ of the poled film was lower than that of the unpoled film at the same temperature, and the primary dispersion of the former appeared at a higher temperature than that of the latter. The externally applied electric field may induce the reorientation of the dipoles not only in the crystalline region (refer to the next section) but also in the disordered amorphous phase.

Effects of Adsorbed Water. The effects of adsorbed water on dielectric properties of specimen were investigated. Figure 6 shows the results of a dried $\alpha 11$ film $\left(10^{-3}\right.$ Torr, $\left.80^{\circ} \mathrm{C}, 12 \mathrm{~h}\right)$ and an undried $\alpha 11 \mathrm{film}$. It was found that the dielectric constant $\varepsilon^{\prime}$ of the undried film is higher than that of the dried film. The relaxation peak of $\varepsilon^{\prime \prime}$ decreased in 


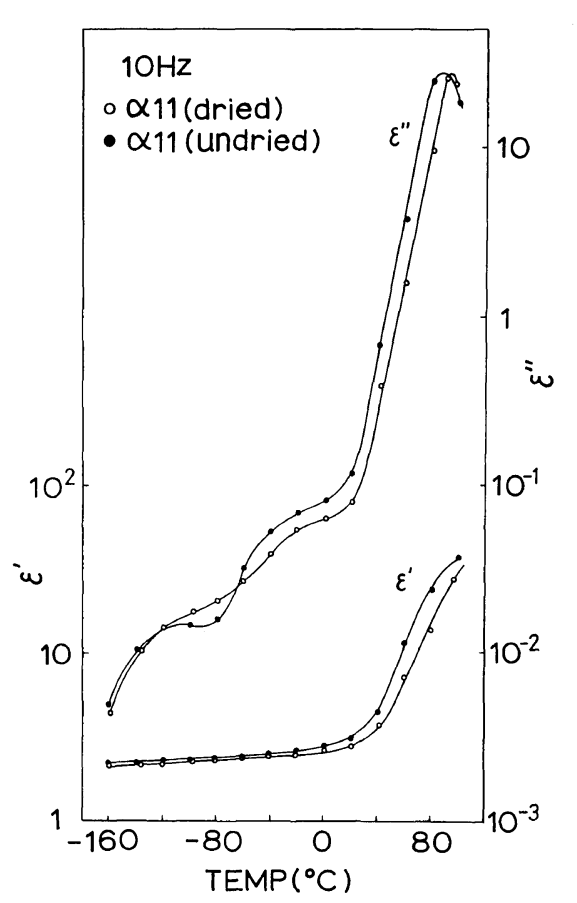

Figure 6. Temperature dependence of dielectric constants of dried and undried specimens.

height and the $\alpha$ peak shifted to the higher temperature side when the film was dried. Figure 7 shows the dependence of dielectric constants on adsorbed water content for $\alpha 9$ and $\alpha 11$ at $20^{\circ} \mathrm{C}$ and $10 \mathrm{~Hz}$. The values of $\varepsilon^{\prime}$ of both $\alpha 9$ and $\alpha 11$ decreased with the evaporation of the adsorbed water. As stated above, the $\beta$ peak has been assigned to the motion of the adsorbed water and the dipole of the amide groups. The increment of the adsorbed water content may increase the number of dipoles in the nylon specimens and the electric conductivity due to the proton motion. ${ }^{12}$ These changes might result in an increase in the dielectric constant of the $\beta$ relaxation. Taking into account the shift of the $\alpha$ peak toward the lower temperature side, it might be better to ascribe these observations to the plasticizing effect of the water; the reorientation of dipoles occurring in the alternating electric field may become easier by such effects.

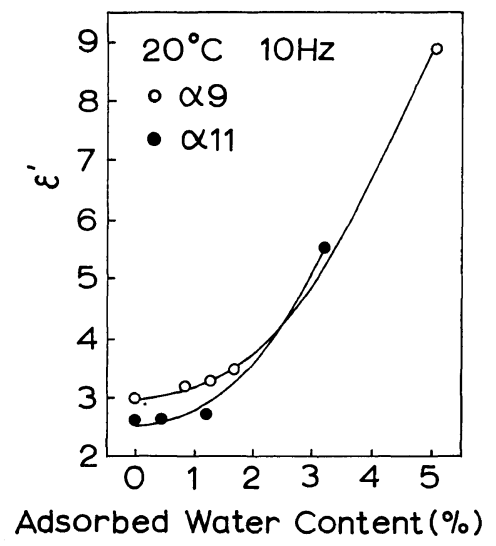

Figure 7. Effects of adsorbed water on dielectric constants of nylon $9(\alpha 9)$ and nylon $11(\alpha 11)$ specimens.

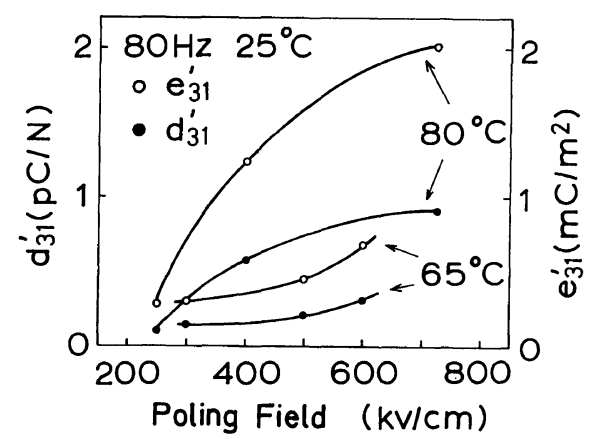

Figure 8. Effects of poling conditions on the piezoelectric constants.

\section{Piezoelectric Measurements}

Anisotropy in the Piezoelectric Constants. The poling-temperature dependence of piezoelectric constants $d_{31}^{\prime}$ and $e_{31}^{\prime}$ in different poling fields has been published for nylon $11,^{2}$ but not for nylon 9 . This dependence for nylon 9 is investigated in this paper. The $\alpha 9$ film was poled at various voltages and temperatures under vacuum for $15 \mathrm{~min}$, and then the piezoelectric constants were measured at room temperature. The results are shown in Figure 8. The highest value of $e_{31}^{\prime}$ was obtained in the highest electric field and at the highest temperature in this experiment. But, because the dielectric breakdown voltage is diverse for each specimen, the poling conditions of $400 \mathrm{kV} \mathrm{cm}^{-1}$ and $80^{\circ} \mathrm{C}$ were adopted in this 


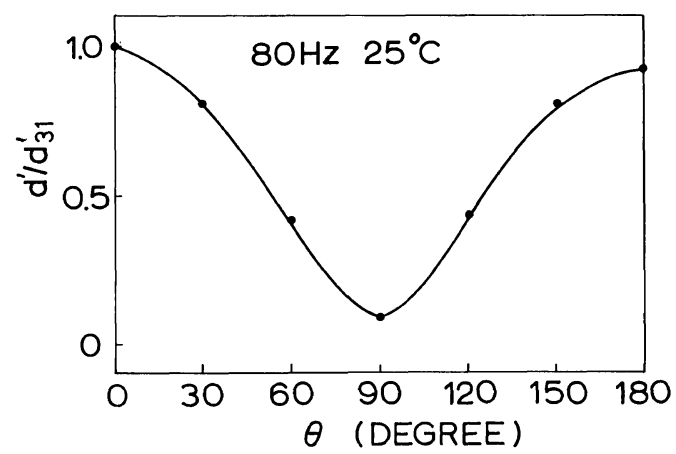

Figure 9. Piezoelecric constant of uniaxially oriented nylon $9 v s$. angle between orientation axis and tensile strain.

work, although not adequate to get the highest piezoelectric constant.

The angle $\theta$ dependence of the piezoelectric strain constant $d^{\prime}$ for the uniaxially stretched and poled specimen $(\mathrm{S}-\alpha 9)$ is shown in Figure 9, where $\theta$ is defined as the angle between the orientation axis and the applied tensile stress. In this figure, the ordinate expresses the ratio of piezoelectric constants $d^{\prime}$ to the maximum piezoelectric constant $d_{31}^{\prime}$. As seen from Figure 9, the maximum piezoelectric constant $\left(d_{31}^{\prime}\right)$ was obtained when the tensile stress was applied to the film in the extended direction, while the minimum piezoelectric constant $\left(d_{32}^{\prime}\right)$ was obtained when the stress was applied to the specimen perpendicularly to the extended direction. The ratio of $d_{31}^{\prime} / d_{32}^{\prime}$ was about 10. The same result was found in the case of nylon 11 . The shape of the curve $d^{\prime} v s . \theta$ and the ratio $d_{31}^{\prime} / d_{32}^{\prime}$ in odd nylon specimens are very similar to those of the oriented poly(vinylidene fluoride) (PVDF) form I film. ${ }^{13}$

Effects of the Adsorbed Water. Figure 10 shows the temperature dependences of the piezoelectric stress constants $e_{31}^{\prime}, e_{31}^{\prime \prime}$ and the piezoelectric strain constants $d_{31}^{\prime}, d_{31}^{\prime \prime}$ for the poled $\alpha 9$ film. The piezoelectric constants did not change so much in the low temperature range, but they increased rapidly above room temperature. The notable peaks were observed
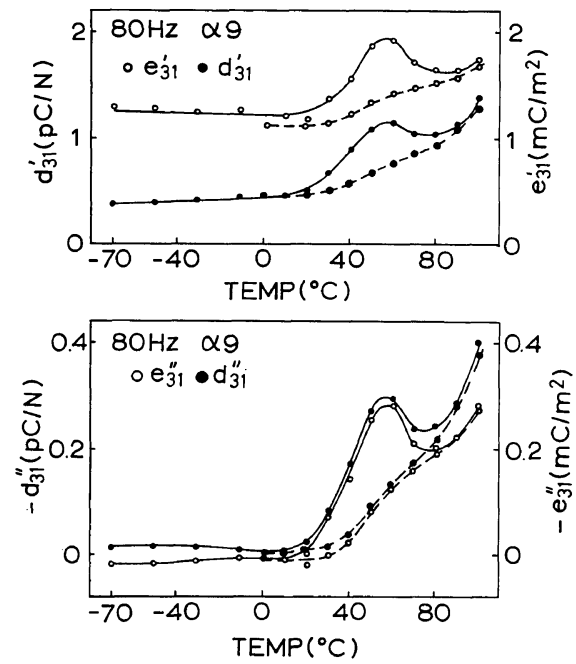

Figure 10. Temperature dependence of piezoelectric constants of the nylon $9(\alpha 9)$ film.

in the temperature range from $50^{\circ} \mathrm{C}$ to $60^{\circ} \mathrm{C}$. When the specimen was cooled from $100^{\circ} \mathrm{C}$ to $0^{\circ} \mathrm{C}$ and the piezoelectric constants were measured again with increasing temperature, the peaks were almost eliminated as shown by the broken lines. After the specimen was left for a few days at room temperature, the peak of the piezoelectric constant appeared again. Therefore, it is considered that the peak is related to the presence of the adsorbed water. At the same time, the temperature dependence of the elastic modulus was also measured and the results are shown in Figure 11. A decrease in elastic constant $E^{\prime}$ and an increase in the piezoelectric constant $d_{31}^{\prime}$ can be seen in the same temperature range of $20-60^{\circ} \mathrm{C}$. In the temperature range from $60^{\circ} \mathrm{C}$ to $80^{\circ} \mathrm{C}, d_{31}^{\prime}$ decreased although the elastic modulus did not vary. In the second heating process mentioned above, a rapid decrease in the elastic modulus at $20^{\circ} \mathrm{C}$ to $40^{\circ} \mathrm{C}$ changed to a gradual decrease from $20^{\circ} \mathrm{C}$ to $80^{\circ} \mathrm{C}$. This observation may also suggest a plasticizing role of water. In Figure $12, e_{31}^{\prime}$ and $d_{31}^{\prime}$ are plotted as functions of adsorbed water content.

All these experimental results of Figures $10-12$ suggest the importance of the adsorbed 


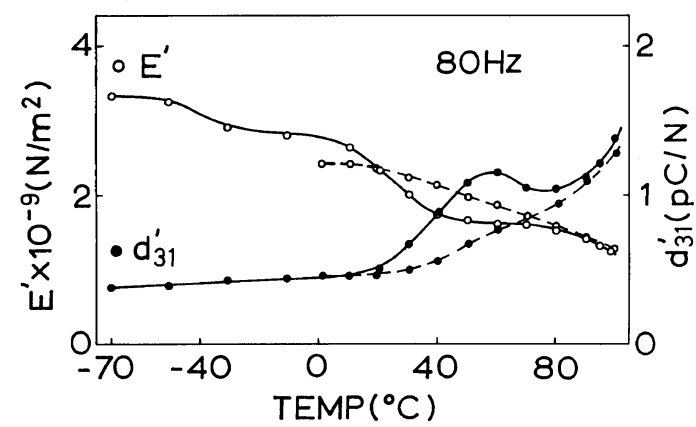

Figure 11. Temperature dependence of elastic modulus and piezoelectric strain constant of the nylon 9 $(\alpha 9)$ film.

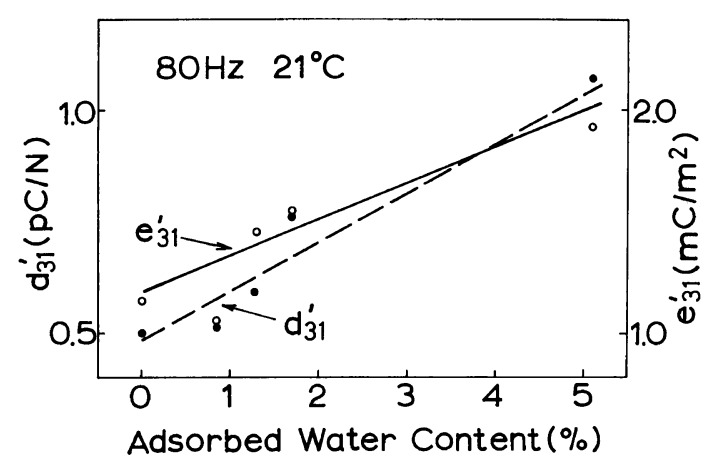

Figure 12. Effects of adsorbed water on piezoelectric constants of the nylon $9(\alpha 9)$ film.

water for the piezoelectric effect of nylon specimens. In the case of the PVDF form I specimen, a heterogeneous effect between the amorphous and crystalline phases has been pointed out to be important for the macroscopic piezoelectric effect. ${ }^{14-16}$ The adsorbed water in the amorphous part of the nylon specimen, by plasticizing effect, decreases the elastic modulus of the amorphous phase and increases the dielectric constant at the same time. Thus differences in the elastic modulus and dielectric constant between the amorphous and crystalline phases become much larger than the dried specimen and the piezoelectric effect becomes larger. Above $50^{\circ} \mathrm{C}$, however, by evaporation of water from the specimen, an increase in $d_{31}^{\prime}$ changes to a decrease in $d_{31}^{\prime}$ resulting in an apparent peak

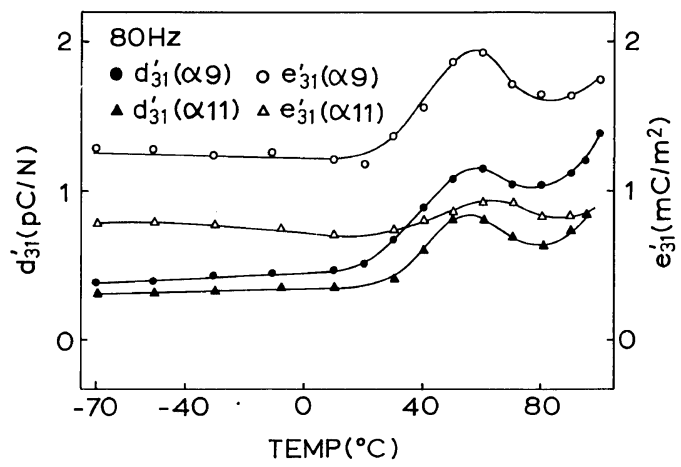

Figure 13. Temperature dependence of piezoelectric constants of nylon $9(\alpha 9)$ and nylon $11(\alpha 11)$ films.

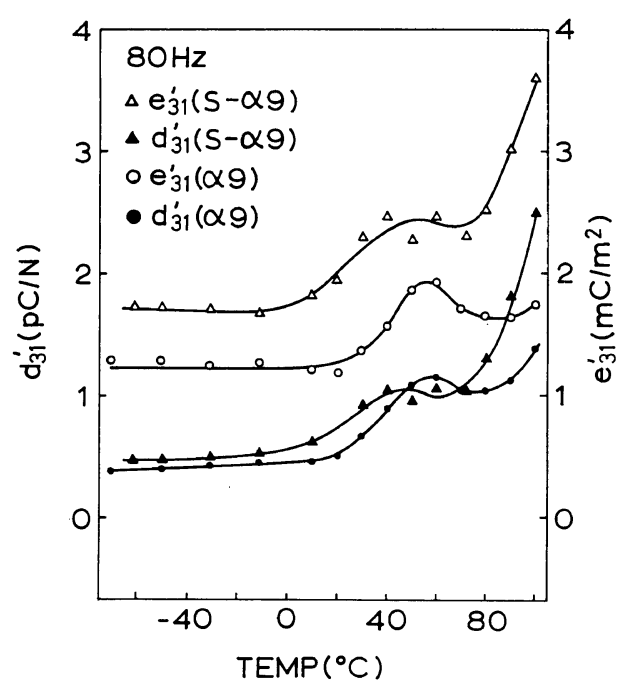

Figure 14. Temperature dependence of piezoelectric constants of stretched and unstretched nylon 9 films.

of $d_{31}^{\prime}$ near $60^{\circ} \mathrm{C}$ as shown in Figure 11. The variation of the elastic modulus may also be a reflection of such water evaporation.

Comparison of the Piezoelectric Constants between nylons 9 and 11. A comparison between piezoelectric constant of $\alpha 9$ and $\alpha 11$ is shown in Figure 13. It is clear that nylon 9 has a larger piezoelectric constant than nylon 11 . Such a difference may be closely related to the larger dipole density of nylon 9 in the crystalline phase.

Effects of Orientation. As shown in Figure 14 , the effects of stretching on the piezoelectric 


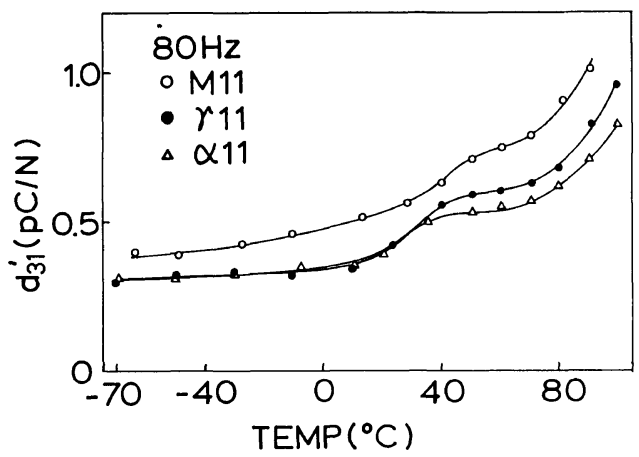

Figure 15. Temperature dependence of piezoelectric constants of M11, $\alpha 11$, and $\gamma 11$ films.

constants were investigated by comparing the two cases of the stretched and poled nylon 9 film (S- $\alpha 9)$ and the unstretched but poled film $(\alpha 9)$. There was no notable change in the piezoelectric strain constant $d_{31}^{\prime}$ between before and after stretching the films, but the piezoelectric stress constant $e_{31}^{\prime}$ increased by the stretching, i.e., 1.3 and $2.1 \mathrm{mC} \mathrm{m}^{-2}$ at $25^{\circ} \mathrm{C}$ for the unstretched and stretched films, respectively. The same appearance was observed also in the case of nylon 11. The increse in the $e$ constant may be owing to the increase in the elastic modulus along the draw direction by the stretching, since the $e$ constant is given by $d \times E$.

Piezoelectric Effect in the $\gamma$ Form. The two poled specimens (M11 and $\gamma 11)$ containing the $\gamma$ form were used for the piezoelectric measurement. Figure 15 shows the comparison of piezoelectric constants among the M11, $\alpha 11$, and $\gamma 11$ films. The M11 film has a higher piezoelectric constant than the $\alpha 11$ film, in good agreement with the results described in the published paper, ${ }^{2}$ while the piezoelectric constant of the $\gamma 11$ film is rather similar to that of the $\alpha 11$ film. In other words, the $\gamma$ phases of the two types of films, M11 and $\gamma 11$, showed different piezoelectric effects from each other. These results may reflect some difference in the crystalline state of the $\gamma$ phase between M11 and $\gamma 11$, even if both films have apparently the same pseudohexagonal lattice. Some au-

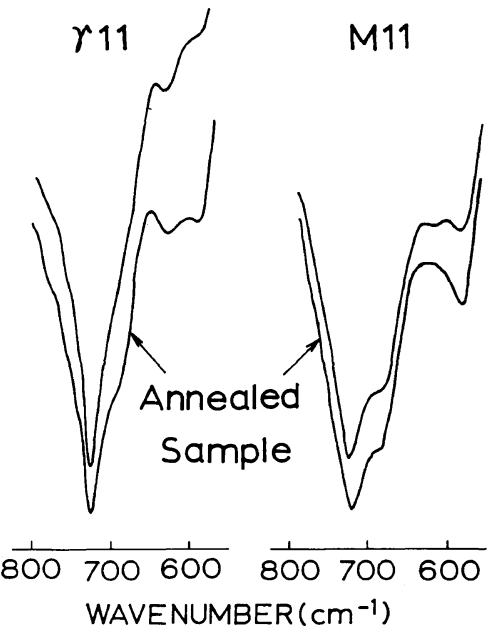

Figure 16. Comparison of infrared spectra between annealed and unannealed $\gamma 11$ and M11 films. The annealing conditions: $120^{\circ} \mathrm{C}, 2 \mathrm{~h}$ under vacuum.

thors proposed that the $\gamma$ structure obtained by the quenching method consists of the disordered packing of the molecular chains with their dipole moment arranged at random around the chain axis of the crystallite. ${ }^{1,6}$ On the other hand, the $\gamma$ phase in the $\gamma 11$ film has a more regular arrangement of the dipole groups and so effective hydrogen bonding is possible between them, considering from the specimen preparation conditions, i.e., crystallization under very slow evaporation of the trifluoroacetic acid solution. Such a difference in the regularity of the $\gamma$ structure between M11 and $\gamma 11$ specimens may reflect on the thermal stability of the $\gamma$ crystalline phase. In fact, on annealing at $120^{\circ} \mathrm{C}$, the $\gamma$ phase of the M11 specimen was easily transformed to the $\alpha$ phase but the $\gamma$ phase of the $\gamma 11$ specimen was not influenced so much, as understood from the infrared spectral data shown in Figure 16. Thus it might be considered that the lower regularity in the dipole orientation within the $\gamma$ crystallite of the M11 specimen makes it relatively easier to rearrange the dipoles in parallel to the electric field in the poling process resulting in the higher piezoelectric effect than that 
Table II. Piezoelectric constants of various specimens at $25^{\circ} \mathrm{C}$

\begin{tabular}{lcc}
\hline $\begin{array}{c}\text { Specimen } \\
\text { code }\end{array}$ & $\begin{array}{c}\text { Piezoelectric stress } \\
\text { constant } e_{31}^{\prime} / \mathrm{mC} \mathrm{m}^{-2}\end{array}$ & $\begin{array}{c}\text { Piezoelectric strain } \\
\text { constant } d_{31}^{\prime} / \mathrm{pC} \mathrm{N}^{-1}\end{array}$ \\
\hline$\alpha 9$ & 1.3 & 0.6 \\
$\alpha 11$ & 0.7 & 0.4 \\
$\gamma 9$ & - & - \\
$\gamma 11$ & 0.7 & 0.4 \\
$\mathrm{M} 9$ & 1.8 & 1.1 \\
$\mathrm{M} 11$ & 1.0 & 0.6 \\
$\mathrm{~S}-\alpha 9$ & 2.1 & 0.8 \\
$\mathrm{~S}-\alpha 11$ & 1.3 & 0.5 \\
$\mathrm{~S}-\gamma 11$ & 1.5 & 0.5 \\
\hline
\end{tabular}

of the $\gamma 11$ specimen.

The piezoelectric constants for all the specimens in the present study are listed in Table II. The largest piezoelectric stress constant $e_{31}^{\prime}$ was obtained from the stretched nylon $9 \alpha$ form film (S- $\alpha 9), 2.1 \mathrm{mC} \mathrm{m}^{-2}$ at room temperature, and the largest piezoelectric strain constant $d_{31}^{\prime}$ was obtained from the mixture of $\alpha$ and $\gamma$ forms of nylon $9,1.1 \mathrm{pC} \mathrm{N}^{-1}$ at room temperature.

\section{CONCLUSIONS}

The dielectric behavior of nylon 9 is similar to that of nylon 11. The dielectric constants of the various specimens were about $2.5-3.0$ at $10 \mathrm{~Hz}$ at room temperature. They increased with increasing temperature, and reached above 30 at about $100^{\circ} \mathrm{C}$. They showed three dielectric loss peaks which originated from the two types of local mode motions $(\gamma$ and $\beta$ ) and the glass transition $(\alpha)$. The value and peak position of the dielectric constant varied by the presence of the adsorbed water as well as the poling condition.

By the piezoelectric measurements of the various kinds of specimens of nylons 9 and 11 , the following characteristic points were clarified.
The nylon 9 films showed a larger piezoelectric effect than nylon 11. This is owing to the dipole density in crystalline phase. It can be expected that the odd nylons of the smaller number of methylene sequences, e.g., nylon 7 , nylon 5, and so on, will show the larger piezoelectric effect through the ideal poling treatment because they have the higher dipole density in the crystallite.

The effects of the adsorbed water on the piezoelectric constant was clarified; the adsorbed water acts as a kind of plasticizer and increases the piezoelectric effect at high temperature if the water evaporation is not allowed.

The melt-quenched specimen consisting of the mixture of $\alpha$ and $\gamma$ forms showed a larger piezoelectric constant than the pure $\alpha$ and $\gamma$ forms under the same poling conditions, because of the difference in the orientational behavior of dipole moments in the crystallites in the poling field.

The correlations among the piezoelectric effect, the dielectric property and the elastic modulus indicated that the piezoelectric effect for the odd nylons can be also interpreted in terms of the difference in mechanical and electrical properties between the amorphous and crystalline phases, as has been pointed out in the case of PVDF form I specimen.

\section{REFERENCES}

1. B. A. Newman, T. P. Sham, and K. D. Pae, J. Appl. Phys., 48, 4092 (1977).

2. B. A. Newman, P. Chen, K. D. Pae, and J. I. Scheinbeim, J. Appl. Phys., 51, 5161 (1980).

3. J. I. Scheinbeim, J. Appl. Phys., 52, 5939 (1981).

4. M. H. Litt, C. H. Hsu, and P. Basu, J. Appl. Phys., 48, 2208 (1977).

5. K. Kato, T. Furukawa, and E. Fukada, Polym. Prep. Jpn., 27, 1800 (1978).

6. V. Gelfandbein and D. Katz, Ferroelectrics, 33, 111 (1981).

7. D. Katz and V. Gelfandbein, J. Phys. D: Appl, Phys., 15, L115 (1982).

8. H. Komoto and K. Saotome, Kobunshi Kagaku, 22, 337 (1965).

9. T. Sasaki, J. Polym. Sci., Polym. Lett., Ed., 3, 557 
Dielectric and Piezoelectric Property of Nylon 9 and Nylon 11

(1965).

10. A. Miyake, J. Polym. Sci., 44, 223 (1960).

11. N. G. McCrum, B. E. Read, and G. Williams, Ed., "Anelastic and Dielectric Effects in Polymeric Solids," John Wiley \& Sons, Inc., New York, N. Y., 1967, Chapter 12.

12. Y. Wada, Ed., "Kobunshi no Kotaibussei," Baifukan, Tokyo, 1971, Chapter 11.

13. Y. Wada, "Piezoelectricity and Pyroelectricity," J.
Mort and G. Pfister Ed., "Electronic Properties of Polymers," John Wiley \& Sons, Inc., New York, N. Y., 1982, Chapter 4.

14. R. Hayakawa and Y. Wada, Rep. Prog. Polym. Phys. Jpn., 19, 321 (1976).

15. H. Tadokoro and K. Tashiro, Kobunshi, 29, 805 (1980).

16. K. Tashiro, M. Kobayashi, H. Tadokoro, and E. Fukada, Macromolecules, 13, 691 (1980). 